\title{
Estratégia para Evitar a Fuga de Conhecimento Organizacional: o caso da ALSTOM Portugal
}

\author{
Raky Wane ${ }^{1}$ \\ Maria João Ferreira Nicolau dos Santos ${ }^{2}$
}

\begin{abstract}
RESUMO
A sobrevivência das organizações depende fortemente da sua capacidade para partilhar e reter conhecimento. Atualmente o risco de perda de conhecimento é elevado, não apenas porque se verifica a saída de grande número de pessoas das organizações por motivo de reforma, dado o envelhecimento da população, mas também porque muitas enfrentam processos de profunda reestruturação ou pertencem a sectores de elevada rotatividade. Uma das ameaças daí decorrentes é a da perda de conhecimento crítico. Situação que poderá pôr em causa a produtividade, competitividade e a capacidade inovadora da organização. Perante este cenário, a melhoria da retenção de conhecimento surge como uma das respostas possíveis para enfrentar o desafio do envelhecimento dos colaboradores no posto de trabalho. No presente artigo, é analisado o caso da unidade fabril da ALSTOM Portugal, onde mais de metade do operadores fabris irá reformar-se nos próximos anos. $\mathrm{O}$ estudo incide sobre o projeto lançado pelo departamento de Gestão de Recursos Humanos (GRH) para potenciar a retenção do conhecimento e responder ao problema da perda de conhecimento organizacional. Analisa, em particular, o processo de identificação do risco de perda de conhecimento crítico e as iniciativas de retenção de conhecimento. Como o projeto ainda se encontra em fase desenvolvimento e de implementação, o estudo de caso recai sobretudo sobre a fase inicial da formulação da estratégia e das propostas de ação para a retenção do conhecimento na unidade fabril da organização.
\end{abstract}

Palavras-chave: gestão do conhecimento, retenção do conhecimento, gestão de recursos humanos; Portugal

\section{Strategy to avoid leaking of organizational knowledge: the ALSTOM Portugal case}

\begin{abstract}
The survival of organizations depends on its capacity to share and retain knowledge. Nowadays the risk of knowledge loss is particularly high. Not only because of retiring, due to aging population, but also because many face processes of deep restructuring or belong to sectors of high turnover. One of the threats resulting is the loss of critical knowledge. This can therefore jeopardise a company's productivity, competitiveness and capacity to innovate. Given this scenario, the improvement of knowledge retention emerges as one of the possible answers to face the challenge of aging employees in the workplace. In this article, we analyse the case of the manufacturing unit of ALSTOM Portugal, where more than half of its factory operators will retire in the next few years. The study is about the project launched by the Human Resources Management department to promote knowledge retention and to tackle the problem of loss of organizational knowledge. It analyses, in particular, the process of identifying the risk of loss of critical knowledge and the initiatives to retain this knowledge. As the project is still under development and implementation, the case study is mainly about the initial phase of the formulation of the strategy and proposals for action to retain knowledge in the manufacturing unit of the organization.
\end{abstract}

Keywords: knowledge management, knowledge retention, human resource management, Portugal

\footnotetext{
${ }^{1}$ Centro de Investigação em Sociologia Económica e das Organizações E Instituto Superior de Economia e Gestão da Universidade de Lisboa - SOCIUS-ISEG-UL.

2- ISEG/UL. Endereço: SOCIUS ISEG/UL, Instituto Superior de Economia e Gestão da Universidade de Lisboa, Rua do Quelhas 6, 1200-781 Lisboa. E-mail: mjsantos@iseg.ulisboa.pt
} 


\section{INTRODUÇÃO}

A evolução demográfica nas sociedades industrializadas tem sido marcada por um acentuado envelhecimento da população e pela saída de grande número de pessoas do mercado de trabalho, nomeadamente por motivo de reforma, o que pode ter enormes implicações no desempenho organizacional. Uma das ameaças mais prementes é, certamente, a da perda de conhecimento crítico para o sucesso organizacional. Situação que poderá pôr em causa a produtividade, competitividade e a capacidade inovadora da organização (e.g. De LONG \& DAVENPORT, 2003; PARISE, CROSS, \& DAVENPORT, 2006). Perante este cenário, a melhoria da retenção de conhecimento, por via da sua partilha, surge como uma das respostas possíveis para enfrentar o desafio do envelhecimento dos colaboradores.

O crescente interesse pela temática tem motivado a realização de diversos estudos. No entanto, poucas foram as investigações que procuraram analisar como as organizações concebem e implementam uma estratégia concertada que responda a este problema. No presente artigo analisamos o caso de uma unidade fabril da ALSTOM Portugal, sucursal do grupo de origem francesa ALSTOM, onde cerca de 70\% dos blue collars (operadores fabris) poderá reformar-se ao longo dos próximos 10 anos.

Analisamos mais especificamente o projeto lançado pelo departamento de Gestão de Recursos Humanos (GRH), o qual procurou responder a três problemas que atualmente afetam a organização: i) o crescente envelhecimento da força de trabalho; ii) a elevada concentração de expertise; e iii) a incapacidade do mercado de trabalho em fornecer blue collars qualificados. Perante os desafios existentes, a empresa avançou com a definição de uma estratégia de retenção de conhecimento. Com o estabelecimento do plano de ação e sua implementação a organização pretende: i) aumentar a produtividade dos blue collars; ii) melhorar a reutilização de conhecimento; iii) acelerar a aprendizagem individual e organizacional e iv) aumentar a flexibilidade organizacional.

O projeto encontra-se ainda em fase desenvolvimento e de implementação, pelo que a análise do estudo de caso recai sobre a fase inicial da formulação da estratégia de retenção de conhecimento. Com o objetivo de perceber como se desenvolveu o processo de concepção e de implementação de uma estratégia de retenção de conhecimento, formulou-se como objetivos para este artigo: (1) analisar o processo de identificação do risco de perda de conhecimento crítico para o funcionamento da unidade fabril em estudo; (2) descrever as 
iniciativas previstas no sentido de se implementar uma estratégia de retenção de conhecimento.

O presente artigo está estruturado em duas partes. Na primeira parte apresentamos o enquadramento teórico-conceptual, no qual contextualizamos o problema da perda de conhecimento e apresentamos um modelo conceptual que agrega quatro dimensões que são consideradas essenciais para a implementação de uma estratégia de retenção de conhecimento. Na segunda parte apresentamos o caso da ALSTOM Portugal com base no modelo conceptual proposto. A partir dos dados recolhidos por meio de uma entrevista em grupo e numa análise documental, analisamos o processo de identificação do risco de perda de conhecimento e apresentamos a estratégia e iniciativas definidas pela empresa para potenciar a retenção do conhecimento. Terminamos o artigo apresentando as principais conclusões extraídas do estudo e identificamos algumas limitações que lhe estão associadas.

\section{ENQUADRAMENTO TEÓRICO-CONCEPTUAL}

No atual enquadramento socioeconómico, a sobrevivência das organizações, independentemente do seu sector de atividade, é fundamentalmente condicionada pela capacidade de combinar conhecimento atualizado e diversificado e, posteriormente, de o incorporar nos serviços e/ou produtos que coloca no mercado. Esta visão remete-nos para a centralidade da gestão do conhecimento (GC) e, em particular, para a importância da partilha e da retenção de conhecimento.

O problema da perda de conhecimento associado ao envelhecimento da população ativa foi analisado por vários autores e.g. ASHWORTH, 2006; SCHMITT, BORZILLO, \& PROBST, 2011). Em 2004, DELONG publicou um dos livros mais referenciados neste domínio, onde identificou, com base em vários exemplos concretos, os impactes organizacionais resultantes da perda de capital intelectual. Para este autor a perda de conhecimento crítico traduz-se numa "diminuição da capacidade de ação eficaz ou da capacidade de tomada de decisão num contexto organizacional específico" (DELONG, 2004, p. 21).

Autores como STRACK e colegas (2008) associaram igualmente o envelhecimento da população ativa ao risco perda de capacidade (capacity risk no original). Neste caso é destacado a perda de conhecimento e expertise, a qual pode afetar a capacidade da empresa em continuar a oferecer determinado produto ou serviço. Acrescentaram, ainda, o risco de 
diminuição da produtividade (productivity risk no original), o qual poderá resultar da perda de força física (importante para operadores fabris, por exemplo), da redução da motivação, devido à diminuição de oportunidades de desenvolvimento de carreira, ou da dificuldade de atualização de competências.

Perante a ameaça de perda de conhecimento crítico importa perceber como as organizações poderão enfrentar este desafio. Analisada a literatura sobre o problema da perda de conhecimento, publicada em revistas científicas ou em revistas especializadas em gestão, verificámos que até à data os estudos têm-se desenvolvido em torno de três grandes áreas: (1) fatores organizacionais que facilitam ou impedem a retenção do conhecimento (e.g. FIEDLER \& WELPE, 2010; MARTINS \& MEYER, 2012); (2) impactes organizacionais resultantes da perda de conhecimento (e.g. HOLAN \& PHILLIPS, 2004; MASSINGHAM, 2008); e (3) iniciativas/ práticas para melhorar a retenção e partilha de conhecimento (e.g. CATTANI, DUNBAR, \& SHAPIRA, 2013; NELSON \& MCCANN, 2010; SCHMITT et al., 2011).

Em conjunto as investigações efetuadas suportam a ideia de que tanto as variáveis de contexto organizacional, tais como estrutura organizacional ou liderança, como as iniciativas que visam o armazenamento e partilha de conhecimento influenciam a memorização e a retenção de conhecimento organizacional. Por exemplo, FIEDLER E WELPE (2010) verificaram que a formalização e a especialização, duas componentes da estrutura organizacional, têm efeitos na retenção de conhecimento. Os autores observaram que a formalização favorece a explicitação do conhecimento, através de manuais, documentação, etc., o que por sua vez tem efeitos positivos em termos de memória organizacional. Por outro lado, as iniciativas que estimulam os fluxos/ partilha de conhecimento também tiveram efeitos positivos na memória organizacional.

Resultados similares foram apresentados por CATTANI e colegas (2013) no que toca à relação positiva entre personalização do conhecimento e retenção de conhecimento ao longo do tempo. A partir de uma análise histórica acerca da construção de instrumentos de corda (ex. violino), na região de Cremona, observou-se que o conhecimento era partilhado e retido através de comunidades de prática (CoPs), onde os contatos e interações constantes entre mestres e aprendizes estimulavam uma aprendizagem com base na observação. Se por alguma razão a relação entre mestre e aprendiz fosse interrompida, a comunidade mitigava o risco de perda de conhecimento crítico, relacionado com a construção dos instrumentos, assumindo-se ela própria como um repositório de conhecimento tácito. 
Apesar dos vários estudos conduzidos, e do seu importante contributo, foi com alguma surpresa que verificámos que poucas investigações empíricas analisaram estratégias de retenção de conhecimento implementadas em contexto organizacional. Existem, contudo, algumas excepções ao nível de frameworks teóricas concebidas a partir de experiências de organizações como a NASA ou a Delta Air Lines, entre outras. Seguidamente, analisam-se dois modelos concebidos para orientar as organizações na implementação de uma estratégia de retenção de conhecimento.

DELONG (2004) foi um dos primeiros a abordar o tema e propôs uma framework que cumpre um duplo propósito. Por um lado, permite auditar e avaliar os sistemas, políticas e práticas em vigor na organização e que intencionalmente, ou não, afetam a retenção de conhecimento. Por outro lado, permite, também, apoiar a identificação e priorização de futuras iniciativas. A framework foi desenvolvida de modo a articular diferentes perspectivas de análise presentes na organização. Do ponto de vista estratégico existe a necessidade de perceber até que ponto a potencial perda de conhecimento afeta a capacidade da organização em prosseguir a sua estratégia. Em termos operacionais interessa analisar os impactes da perda de conhecimento nas operações (por exemplo, em que medida a perda de conhecimento sobre os clientes afeta diretamente as vendas). Ao nível da perspectiva de GC interessa, essencialmente, desenvolver uma base de conhecimento única e distintiva. Por fim, a visão de GRH valoriza a criação de um ambiente organizacional "amigo" do conhecimento. Com base neste racional a framework proposta aponta quatro tipos de iniciativas interdependentes: (1) práticas de transferência de conhecimento; (2) ferramentas informáticas para capturar, armazenar e partilhar conhecimento; (3) práticas e processos de $\mathrm{RH}$; e (4) iniciativas de recuperação de conhecimento.

Mais recentemente, LIEBOWITZ (2009) propôs outra framework que visa apoiar as organizações na implementação de uma estratégia de retenção de conhecimento. Segundo o autor esta estratégia deverá assentar em quatro pilares centrais: (1) reconhecimento e recompensa; (2) fluxos de conhecimento intergeracionais (bidirecionais); (3) personificação e codificação de conhecimento; e (4) manutenção do relacionamento com colaboradores reformados (Golden Gem, no original).

O pilar relativo ao reconhecimento e recompensas realça a necessidade de integrar as atividades que visam a retenção do conhecimento na rotina diária dos colaboradores, motivando-os a participar ativamente nas mesmas. A partilha de conhecimento entre gerações é outro dos pilares considerados críticos. O terceiro tipo de iniciativas relaciona-se com o 
anterior, destacando a necessidade de conciliar a codificação e personalização enquanto estratégias de transferência e partilha de conhecimento. Por fim, o Golden Gem enfatiza a importância de se estabelecer uma relação com os colaboradores que vá para além da sua permanência na empresa.

LIEBOWITZ (2009) e DELONG (2004) realçaram também a importância de se perceber, previamente, qual o conhecimento que a organização tem interesse em reter. Com efeito, "o primeiro passo para a retenção do conhecimento [organizacional] crítico consiste em identificar que conhecimento essencial está em risco de ser perdido (DE LONG \& DAVENPORT, 2003, P. 51)". Por exemplo, se um colaborador vai sair da empresa é importante perceber se o conhecimento que ele detém é crítico, se existe um sucessor dentro da organização e de que forma o conhecimento dessa pessoa poderá ser partilhado e memorizado pela organização.

Apesar das componentes sugeridas apresentarem designações distintas, e da framework proposta por LIEBOWITZ (2009) ser provavelmente menos abrangente, em nosso entender, as semelhanças entre elas são bastante óbvias. Ambos os modelos reconheceram que o sucesso da estratégia de retenção passa, em grande medida, pelo envolvimento ativo dos colaboradores seniores e juniores. Envolvimento este que requer por um lado, a criação de oportunidades (ou canais) para a circulação de conhecimento e, por outro lado, que as pessoas estejam dispostas a alimentar e desbloquear esses fluxos de conhecimento. Isto significa que, para além das questões da partilha de conhecimento intergeracional, também a motivação e outras políticas que tipicamente se associam à área de GRH (ex. planos de sucessão) devem ser consideradas aquando da formulação de uma estratégia de retenção de conhecimento. Por outro lado, os autores destacaram, também, que a organização possui múltiplas tipologias de conhecimento e nem todo tem a mesma relevância estratégica. Razão que terá levado os dois autores a defender que o primeiro passo na formulação de uma estratégia de retenção de conhecimento deverá ser a identificação do risco de perda do conhecimento crítico para o sucesso organizacional.

Após a análise de estudos empíricos, bem como das frameworks teóricas acima referidas, verificamos que existem quatro componentes centrais a considerar na formulação de uma estratégia de retenção de conhecimento: (1) risco de perda de conhecimento; (2) infraestrutura organizacional de retenção de conhecimento; (3) iniciativas de partilha e armazenamento de conhecimento; e (4) políticas de desenvolvimento e retenção de pessoas. Em nosso entender estas componentes refletem a necessidade de conciliar as perspetivas 
estratégica e operacional, bem como as perspetivas de $\mathrm{GC}$ e $\mathrm{RH}$, tal como sugerido por DELONG (2004).

A figura 1 ilustra as várias componentes, bem como a interligação entre as mesmas (representada na figura através das setas). Nos pontos seguintes analisamos de forma mais desenvolvida cada uma das componentes.

Figura 1 - Componentes a considerar na formulação de uma estratégia de retenção de conhecimento

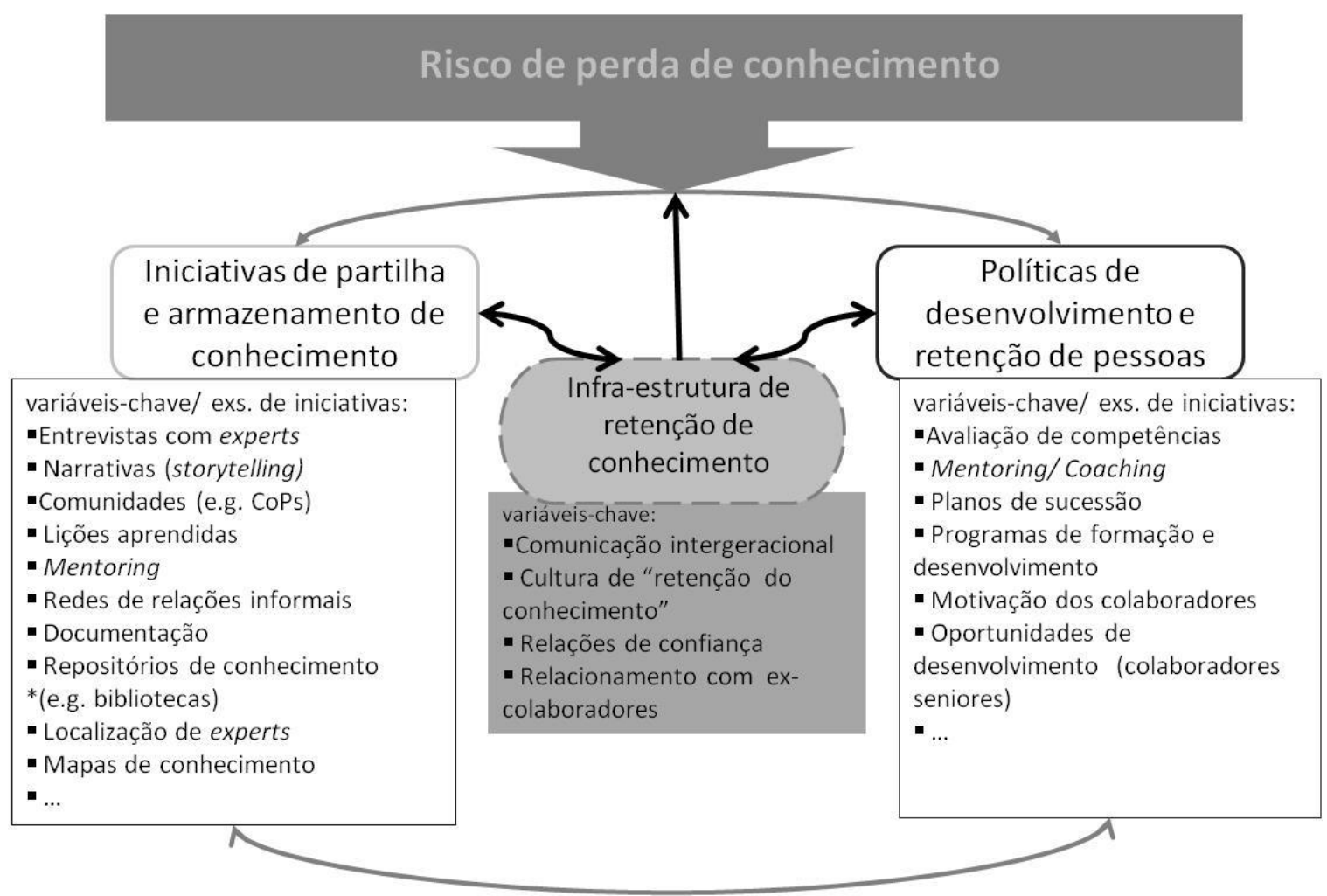

Fonte: os autores

\section{A. Identificação do conhecimento em risco}

Considerando que a perda de conhecimento tem consequências negativas, importa avaliar risco de perda de conhecimento na organização (DE LONG, 2002; LIEBOWITZ, 2009 E STRACK et al., 2008). Para esse efeito, vários autores propuseram a utilização de métricas que, perante a saída dos colaboradores, dão indicação sobre o risco de perda de conhecimento organizacional crítico (por exemplo, multiplicando o númerode anos que faltam para a reforma por uma estimativa da importância do colaborador no funcionamento operacional da empresa (DE LONG, 2002) ou ainda sinalizando, de entre os colaboradores 
que vão deixar a empresa, qual a potencial perda de conhecimento crítico (DE LONG \& DAVENPORT, 2003).

Ainda assim estas abordagens não são isentas de críticas. Alguns autores consideram que a simples identificação do conhecimento em risco na organização não é suficiente para garantir que o conhecimento crítico é retido. PARISE e colegas (2006), por exemplo, referem que é igualmente necessário identificar quem possui esse conhecimento. Para estes autores estas abordagens apresentam dois grandes problemas. Por um lado, não permitem capturar o conhecimento na íntegra, como é o caso, por exemplo, das razões que estiveram na origem de uma boa decisão no passado. Por outro lado, nem sempre têm em consideração as relações sociais implícitas na concretização de determinada tarefa/função. No entender destes autores, a solução para estas duas questões passa por empregar uma metodologia de análise de redes sociais de forma a atingir um duplo objetivo: (1) identificar as vulnerabilidades criadas pela perda de conhecimento técnico e relacional e (2) conceber estratégias de retenção de conhecimento ajustadas ao papel que o colaborador ocupa na rede - elo central, periférico ou broker (PARISE et al., 2006).

Analisando os vários autores, concluímos que seja qual for a abordagem empregue técnicas tradicionais ou metodologia de análise de redes sociais - interessa, acima de tudo, perceber se o conhecimento que permanece na organização lhe permite concretizar as suas metas estratégicas. Isto pressupõe: i) identificar previamente qual é o conhecimento crítico para o desempenho organizacional; ii) avaliar o risco de perda de conhecimento (recorrendo a indicadores como a média de idades dos colaboradores ou a relevância do conhecimento em risco, entre outros); e iii) avaliar os impactes associados a esse risco, como por exemplo, o risco de capacidade e o risco de produtividade (STRACK et al., 2008). No caso de se concluir, após esta análise, que a capacidade da organização ficará afetada é altura de desenvolver esforços no sentido de reter o conhecimento que se considerou crítico.

\section{B. Infraestrutura organizacional para a retenção de conhecimento}

Grande parte do conhecimento organizacional relevante reside nas pessoas. Este é partilhado por meio de relacionamentos pessoais e/ou institucionalizado através de componentes estruturais, tais como processos, rotinas, meios informáticos, etc. (SANTOS, WANE, \& LOPES, 2014). Por isso, além de iniciativas que estimulem os fluxos de conhecimento ou o desenvolvimento dos colaboradores, existe toda uma infra-estrutura organizacional que afeta a retenção de conhecimento. 
A ausência de relação interpessoal, a falta de competências de relacionamento e comunicação, assim como a ausência de confiança entre as pessoas são alguns dos aspetos que podem obstaculizar os fluxos de conhecimento (CHRISTENSEN, 2007; RIEGE, 2005) e, consequentemente, os esforços de retenção de conhecimento. Ao colocarmos este componente no centro da formulação da estratégia pretendemos, sobretudo, chamar a atenção para a necessidade de se desenvolver um ambiente organizacional "amigo da retenção do conhecimento". De entre os elementos que integram esta infa-estrutura organizacional destacamos três fatores em particular: i) a cultura organizacional; ii) a infra-estrutura comunicacional e iii) a confiança.

As investigações realizadas têm demonstrado que a cultura influencia fortemente $o$ comportamento dos membros da organização no que toca ao desenvolvimento do conhecimento (e.g. DE LONG \& FAHEY, 2000; MCDERMOTT \& O’DELL, 2001). Ao nível da retenção de conhecimento é fundamental que a organização promova nos colaboradores a vontade (willingness) de partilhar conhecimento, assim como o hábito de procurar e de reutilizar o conhecimento existente na organização (DELONG, 2004).

Todavia, quando se trata de questões culturais não existe uma fórmula única para o sucesso, nem um conjunto de melhores práticas. Vários autores defendem que as iniciativas orientadas para a melhoria dos fluxos de conhecimento devem ser selecionadas em função da cultura organizacional existente, utilizando os canais de comunicação e as redes sociais previamente construídas (MCDERMOTT \& O’DELL, 2001; RIEGE, 2005).

É através dos canais de comunicação e das redes sociais que os membros da organização partilham ideias, trocam experiências ou procuram/ recebem informação que consideram útil (SANTOS, WANE \& LOPES, 2014). Fatores que, em última instância, poderão reforçar a motivação dos colaboradores para se tornarem agentes ativos nestes processos. A comunicação virtual pode facilitar o armazenamento e disseminação do conhecimento pela organização. A tecnologia pode melhorar a retenção de conhecimento, na medida em que favorece a comunicação intergeracional (aproximação entre colaboradores seniores e juniores), a aprendizagem colaborativa, a captura de conhecimento (documentação, bases de dados, etc.) e o mapeamento do conhecimento (DELONG, 2004). A comunicação presencial, por seu lado, incentiva o diálogo e a partilha de emoções, experiências e modelos mentais (NONAKA \& KONNO, 1998). O que contribuirá para promover o apoio/ colaboração e a confiança entre os membros seniores e juniores. 
No caso das relações de confiança, estas constroem-se ao longo do tempo, com base na comunicação interpessoal e nas interações sociais (NAHAPIET \& GHOSHAL, 1998). A confiança é muitas vezes referida como um dos principais fatores facilitadores/ inibidores dos fluxos de conhecimento (CHRISTENSEN, 2007; RIEGE, 2005; SUN \& SCOTT, 2005). O racional subjacente é o de que os níveis de confiança existentes influenciam as decisões dos membros da organização quanto ao seu envolvimento nos processos de partilha de conhecimento e facilitação da aprendizagem (IPE, 2003). Talvez por isso exista alguma sobreposição entre as práticas de GC e RH que são sugeridas para reforçar os níveis de confiança entre colaboradores, no geral, e entre gerações, em particular. Alguns exemplos são os programas de mentoring, o coaching e a rotação de funções, entre outros (e.g. ARMSTRONG-STASSEN \& SCHLOSSER, 2011; DELONG, 2004; LIEBOWITZ, 2009).

\section{Iniciativas de armazenamento e partilha de conhecimento}

No quadro de uma estratégia de retenção de conhecimento os processos de armazenamento e partilha surgem como veículos para a institucionalização do conhecimento crítico nas rotinas organizacionais. De acordo com WALSH e UNGSON (1991), a informação pode ser armazenada (ou retida) através de: (1) pessoas e suas experiências; (2) cultura organizacional por meio de símbolos, linguagem e artefactos; (3) práticas e procedimentos de trabalho; (4) estrutura e papéis organizacionais; e (5) "ecologia" do contexto de trabalho (i.é. na estrutura física dos postos de trabalho).

Nas duas frameworks analisadas, assim como em grande parte das investigações empíricas revistas, as iniciativas propostas em termos de transferência de conhecimento visam a codificação ou a personalização do conhecimento (e.g. FIEDLER \& WELPE, 2010; LIEBOWITZ, 2009).

Para HANSEN e colegas (1999) - os primeiros a distinguir estratégias de codificação de estratégias de personalização - as organizações tendem a adotar maioritariamente uma das estratégias, sendo que a seleção da mesma varia em função do posicionamento competitivo da empresa. A opção pela codificação pressupõe a adoção de iniciativas como a criação de bases de dados de conhecimento, a generalização das lições aprendidas, a conceção de documentação, a formação profissional e as entrevistas com experts, entre outras ${ }^{3}$ (DELONG, 2004; HANSEN, NOHRIA, \& TIERNEY, 1999; LIEBOWITZ, 2009).

\footnotetext{
${ }^{3}$ Para uma descrição mais exaustiva de algumas das práticas de GC referidas ver CEN, 2004; DELONG, 2004; RAO, 2005.
} 
Caso, o objetivo seja o de fomentar os fluxos de conhecimento, aproximando os detentores do conhecimento para que possam partilhar conhecimento tácito (HANSEN et al., 1999), então a criação de CoPs, o mentoring e coaching, a rotação de funções e a utilização de narrativas, entre outras, surgem como exemplos típicos de práticas de GC implementadas no âmbito de uma estratégia de personalização (DELONG \& DAVENPORT, 2003; DELONG, 2004; LIEBOWITZ, 2009).

Uma outra questão central na implementação deste conjunto de iniciativas prende-se com aquilo que LIEBOWITZ (2009) designou por fluxos de conhecimento bidireccionais. Ou por outras palavras, é importante que os colaboradores seniores partilhem o seu conhecimento com os juniores, ao mesmo tempo que adquirem determinados conhecimentos específicos (ex. conhecimentos tecnológicos). A partilha de conhecimento entre diferentes gerações é uma área que tem vindo a ganhar destaque nos últimos anos. Autores como APPELBAUM e colegas (2012; 2012) e CALO (2008), entre outros, conduziram estudos que suportam a ideia de que os colaboradores seniores têm interesse/motivação em partilhar conhecimento com os colegas juniores. Todavia, é necessário ter em consideração que as motivações, formas de comunicação e as necessidades dos colaboradores variam em função da proximidade da idade de reforma. Assim, a organização deve, acima de tudo, encorajar o espírito de equipa a colaboração entre seniores e juniores, de forma a fortalecer as relações de confiança entre gerações (APPELBAUM, GUNKEL, et al., 2012). Estes princípios deverão, portanto, ser considerados quer na seleção das iniciativas de GC a implementar, quer nas políticas de RH.

\section{Políticas e práticas de desenvolvimento e retenção de pessoas}

De acordo com as teorias de gestão estratégica de RH e de capital intelectual, o argumento subjacente é o de que as práticas de atração, desenvolvimento e retenção de pessoas contribuem para a construção de uma base de conhecimento única e distintiva (CABRERA, COLLINS, \& SALGADO, 2006; MINBAEVA, FOSS, \& SNELL, 2009).

Neste sentido, importa aceder e partilhar o conhecimento que reside nas pessoas, mas também aquele que reside nas suas relações ou está institucionalizado através de processos e rotinas organizacionais (WRIGHT, DUNFORD, \& SNELL, 2001). As políticas e práticas de RH poderão contribuir para este propósito na medida em que promovem as relações sociais e a confiança entre colaboradores, a interação e colaboração, o compromisso organizacional, a interdependência, a equidade e o desenvolvimento do capital humano da organização (WANE \& SANTOS, 2014). Tipicamente, no quadro de uma estratégia de retenção de conhecimento 
as políticas de $\mathrm{RH}$ consideradas incluem iniciativas no âmbito i) do desenvolvimento e carreiras, ii) da motivação e iii) da retenção de colaboradores.

As práticas de desenvolvimento de RH visam dar resposta às necessidades de conhecimento da organização e, em simultâneo, desenvolver o stock de conhecimento, designadamente através da aprendizagem e reutilização/ combinação de conhecimento existente. Primeiramente, importa mapear o conhecimento existente na organização de forma a perceber se o conhecimento necessário está disponível. Entre outras práticas, a organização pode realizar auditorias de conhecimento e/ou implementar sistemas de avaliação de competências (CEN, 2004; DELONG, 2004). Uma vez conhecida a base de conhecimento existente é altura de desenvolver e reter essas competências e conhecimento. Tal pode passar por implementar programas de formação, mentoring, coaching, ou através da rotação de funções, entre outros (APPELBAUM, BENYO, et al., 2012; DELONG, 2004; STRACK et al., 2008).

Ao nível do planeamento de carreiras o que se pretende é, por um lado, minimizar os impactes resultantes da saída de colaboradores chave e, por outro lado, incentivar o desenvolvimento dos colaboradores. Entre as iniciativas mais utilizadas para este fim encontram-se os planos de sucessão. Este planeamento consiste em identificar, simultaneamente, colaboradores cujas competências/conhecimentos necessitam ser substituídas num futuro próximo e aqueles que têm potencial para desenvolver essas mesmas competências/conhecimentos. Neste sentido, "os processos de desenvolvimento de carreira fornecem um road map para o desenvolvimento das capacidades que devem ser transferidas para a geração seguinte (DELONG, 2004, p. 64).”

Conforme já se percebeu a retenção de conhecimento depende, fundamentalmente, do envolvimento dos colaboradores seniores e juniores nos processos de partilha de conhecimento. O que significa que os colaboradores deverão estar (ou ser) motivados para se envolverem ativamente nesse processo. Do ponto de vista motivacional, LIEBOWITZ (2009) destacou dois aspetos essenciais. O primeiro prende-se com o fato de integrar as atividades de retenção de conhecimento na rotina diária da organização. Desta forma espera-se que as pessoas se envolvam neste processo sem a perceção de que estão a fazer um esforço adicional. O segundo aspeto relaciona-se com a necessidade de reconhecer e recompensar o envolvimento dos colaboradores. Por exemplo, uma forma de reconhecer os colaboradores seniores poderá ser através de programas de mentoring em que a organização os reconhece como experts num determinado domínio. 
CALO (2008), por seu lado, associou a motivação dos colaboradores seniores a fatores como o (re)desenho de funções ou o desenvolvimento de carreiras. Assim, considerando que os colaboradores seniores têm motivações e necessidades distintas dos juniores importa refletir essas especificidades nas políticas e práticas de RH (APPELBAUM, BENYO, et al., 2012). Por exemplo, ARMSTRONG-STASSEN \& SCHLOSSER (2011) verificaram que o sentimento de pertença dos colaboradores seniores tem efeitos positivos nas suas decisões de permanecer na organização. Sendo de realçar que o sentimento de pertença destes colaboradores seniores é reforçado quando estão reunidas as seguintes condições: (1) existem práticas de RH consistentes com suas as necessidades e preferências; (2) os gestores de equipa/ supervisores implementam essas práticas de forma justa e equitativa; e (3) existe a perceção, por parte destes colaboradores, que o seu contributo é valioso para a concretização das metas organizacionais.

Ainda a propósito das políticas de RH orientadas para a retenção de colaboradores seniores destacamos uma iniciativa que tem vindo a ser sugerida por vários autores (e.g. APPELBAUM, BENYO, et al., 2012; DELONG, 2004): a opção de faseamento da reforma (phased retirement, nos originais). O faseamento da reforma é uma iniciativa onde se incentiva uma transição gradual para a reforma, ao invés de uma separação abrupta entre o colaborador e a organização (CALO, 2008). De acordo com a Associação Americana de Pessoas Reformadas (AARP), a redução do tempo de trabalho, a partilha de trabalho, a flexibilidade de horários ou a consultoria, são exemplos de iniciativas que podem ser aplicadas a colaboradores com mais de 50 anos de forma a preparar a transição para a reforma (ver AARP, 2006).

\section{ESTUDO DE CASO: UMA ESTRATÉGIA PARA A RETENÇÃO DO CONHECIMENTO DOS BLUE COLLARS}

\section{A. Descrição da empresa}

O projeto, em análise no presente artigo, foi desenvolvido na sucursal portuguesa da ALSTOM e, em particular, na unidade fabril da empresa localizada em Setúbal. O core business desta unidade é a construção soldada e emprega cerca de $58 \%$ do total dos trabalhadores da ALSTOM Portugal. A unidade começou a laborar em 1976, como unidade II da Mague Construções Metalomecânicas S.A., tendo sido responsável pelo fabrico de todas as caldeiras para Centrais Térmicas a Carvão e de Cogeneração instaladas pela EDP em 
Portugal. Constitui atualmente um dos dois centros que o grupo ALSTOM dispõe a nível mundial para o fabrico de caldeiras de recuperação de grande porte (HRSG - Heat Recovery Steam Generators) para centrais de ciclo combinado a gás.

Após o ano de 2009, de modo a fazer face às necessidades do mercado e num esforço de maior flexibilização, bem como devido a uma grande redução na procura de HRSG's, esta unidade começou a produzir condensadores e permutadores de calor (de alta e baixa pressão). Desde então o esforço para a diversificação da produção mantém-se. No sentido de expandir a sua atividade à fabricação de componentes para centrais nucleares, em 2013, foi concluída uma obra de expansão, com a criação de mais um pavilhão, garantindo as condições adequadas para a fabricação dessas mesmas componentes.

\section{B. A Gestão de Conhecimento na ALSTOM}

O carácter eminentemente tecnológico das atividades desenvolvidas pela empresa, associada à sua expansão para diversas áreas geográficas, justificam a importância estratégica da transferência e de retenção de conhecimento na ALSTOM. Todavia, apesar do enfoque ainda existem algumas lacunas ao nível da formalização e da conceção de processos de retenção e de transferência do conhecimento individual. Particularmente, foram identificados problemas no que se refere à transferência do conhecimento e de competências dos colaboradores do fabrico - os blue collars -, nomeadamente em unidades de menor dimensão, como é o caso da ALSTOM Portugal.

Perante esta constatação a ALSTOM Portugal iniciou, em 2013, um projeto interno que visava recolher, organizar e potenciar os conhecimentos técnicos existentes na organização garantindo a sua viabilização no futuro. Para a implementação do projeto foi constituída uma equipa multifuncional que articulou as áreas de gestão de pessoas e as áreas operacionais.

\section{a) Motivações e objetivos do projeto.}

Através deste projeto a ALSTOM Portugal pretende encontrar respostas para os desafios que interna e externamente se lhe colocam. Grande parte dos colaboradores da unidade fabril, em particular os do fabrico (blue collars), são altamente qualificados, no entanto a expertise existente poderá ser posta em causa dada a existência de um elevado número de trabalhadores em idade de reforma. A composição etária da força de trabalho terá sido determinante para que a ALSTOM em Portugal considerasse fundamental a elaboração 
de um planeamento estratégico de $\mathrm{RH}$, no qual a retenção de conhecimento e a preparação de sucessores para estas funções assumem especial relevo.

Um outro aspeto identificado como problemático foi o facto de haver uma grande concentração de expertise, ou seja, determinados conhecimentos técnicos (específicos dos tipos de produto fabricados) encontram-se apenas numa pequena percentagem de trabalhadores. Existe assim a necessidade de transformar o conhecimento maioritariamente técito em conhecimento explícito, i.é. conhecimento materializado, passível de ser transferido, comunicado, armazenado, preservado e compreendido por outros (BERGAMASCHI \& URBINA, 2009; MARTINS, 2010).

Acresce ainda as questões da envolvente externa. Dada a especificidade das atividades desenvolvidas na unidade fabril não existem disponíveis no mercado de trabalho, ou pelo menos não em quantidade suficiente, as competências e conhecimentos requeridos pela empresa. Pelo que, qualquer colaborador recrutado deverá desenvolver um conjunto de competências relacionadas com a especificidade dos processos da empresa, o que é uma tarefa morosa e complexa.

Por fim, há ainda que considerar o facto de o sector de atuação da ALSTOM Portugal exigir uma enorme flexibilidade na organização do trabalho, devido à volatilidade das cargas de trabalho. Esta situação requer que a empresa e os seus RHs respondam de forma rápida, eficaz e eficiente às solicitações de um mercado volátil e aos requisitos, cada vez mais exigentes, dos clientes. Uma exigência que obriga os colaboradores a uma constante adaptação a novas necessidades funcionais.

Confrontada com os desafios acima identificados a empresa considerou que era necessário desenvolver esforços no sentido de potenciar o conhecimento técnico disponível na organização. A nível interno o desafio passa por reter e disseminar a expertise técnica que está atualmente concentrada em poucas pessoas, ou em pessoas com médias de idade acima dos 55 anos. Do ponto de vista externo, o desafio consiste em desenvolver uma base de conhecimento capaz de responder de forma ágil e eficaz às necessidades dos clientes. Neste enquadramento, a ênfase foi colocada nas componentes de retenção e transferência de conhecimento como forma de melhorar o desempenho individual e organizacional. Mais concretamente, a empresa pretende: melhorar a produtividade dos colaboradores; melhorar a reutilização do conhecimento existente; acelerar a aprendizagem individual e organizacional; melhorar a flexibilidade organizacional. 


\section{Metodologia de investigação}

Este estudo pretendeu perceber como se desenrola o processo de formulação e implementação de uma estratégia de retenção que minimize a ameaça de perda de conhecimento. Para explorar a temática optámos por uma metodologia de estudo de caso, no sentido de obtermos informação mais aprofundada sobre a formulação da estratégia de retenção de conhecimento na unidade fabril em estudo. $\mathrm{O}$ estudo de caso é especialmente indicado quando se pretende perceber um fenómeno complexo, permitindo ao investigador captar as características/ significados dos eventos reais - tais como processos organizacionais, entre outros - de forma holística (YIN, 2003).

Encontrando-se o projeto de conceção da estratégia de retenção numa fase inicial, o presente estudo teve como objetivos: (1) analisar o processo de identificação do risco de perda de conhecimento crítico para o funcionamento da unidade fabril em estudo; (2) descrever as iniciativas previstas no sentido de implementar a referida estratégia.

A recolha de dados foi efetuada por meio de uma entrevista em grupo (focus group) realizado nas instalações da organização, com duração de 1 hora e 40 minutos, onde participaram: um conselheiro expert, três responsáveis das áreas de fabrico em estudo e um responsável da engenharia de soldadura. Considerámos que o focus group era ajustado tendo presentes os objetivos anteriormente referidos, na medida em que se pretendia estimular a participação de vários níveis hierárquicos, bem como obter opiniões diversificadas sobre o problema da perda de conhecimento e possíveis soluções neste contexto organizacional. Em comparação com a observação participante ou com as entrevistas individuais, a vantagem do focus group é que permite observar as interações entre os participantes, ou seja, momentos nos quais são expressas as suas atitudes e experiências (MORGAN \& SPANISH, 1984). O conteúdo da entrevista foi, posteriormente, analisado tendo em consideração um conjunto de componentes definidas a priori (ver figura 1).

Os dados recolhidos no focus group foram complementados com a análise de documentação interna. As organizações implementam um conjunto de práticas que influenciam, intencionalmente ou não, a retenção de conhecimento organizacional crítico (DELONG, 2004). Neste sentido, foi analisada a documentação do projeto e outras relacionadas com práticas de gestão de pessoas e do conhecimento. 


\section{Discussão e análise}

A análise dos resultados apresenta-se em dois sub-pontos. Primeiramente, analisamos de que forma os responsáveis das equipas percecionam o risco de perda de conhecimento na unidade fabril em estudo. Seguidamente, apresentamos uma breve descrição dos próximos passos relativos à implementação da estratégia de retenção de conhecimento.

\section{a) Risco de perda de conhecimento.}

A recolha de dados através de focus group cumpriu um duplo propósito: identificar o risco de perda de conhecimento crítico e recolher pistas para a formulação de um plano de ação que permita mitigar esse risco. Analisamos, por agora, a abordagem seguida e os resultados relativos à identificação do risco de perda de conhecimento.

A análise e a avaliação do risco de perda de conhecimento foi efetuada com o recurso a técnicas qualitativas. Tomou-se como referência a análise das perceções dos participantes que integraram o focus group, tendo sido esta informação complementada com dados recolhidos internamente (ex. análise SWOT relativa aos blue collars). Como resultado, foram identificados os perfis profissionais críticos (CALO, 2008) para a concretização dos processos de fabrico, entre os colaboradores próximos da idade de reforma, foram identificados os seus conhecimentos críticos e únicos/ distintivos (DELONG \& DAVENPORT, 2003). Por fim, foram identificados os potenciais impactes decorrentes da perda desse conhecimento (DELONG, 2004; STRACK et al., 2008; MASSINGHAM, 2010).

No âmbito do focus group os participantes identificaram de início os perfis profissionais críticos para a concretização dos processos de fabrico, bem como as necessidades de conhecimento, know-how e competências para o desempenho desses processos. Concluída a análise, foram selecionados dois perfis específicos - doravante designados por perfil A e perfil B - para os quais a retenção de conhecimento foi percecionada como crítica. A afirmação seguinte resume bem a identificação dos perfis chave:

“As competências principais que eu vejo ali [na fábrica] são os [Perfil A] e os [Perfil B] (...). Eles [colaboradores] adquiriram aquelas competências trabalhando com outros que tinham essas competências durante 10/15 anos. (...) Não se consegue transmitir durante 2 ou 3 anos essas competências. (...) Isto é [um conhecimento] demasiado específico e técnico.”

Para estes dois perfis profissionais a ameaça de perda de conhecimento foi amplamente expressa. Como critérios de análise (utilizados pelos participantes do focus group), foram considerados a média de idades dos colaboradores e o tempo necessário para formar profissionais competentes nestes domínios, o qual associado às lacunas do mercado 
em termos da oferta de formação destes perfis, torna real a potencial perda de conhecimento crítico na organização. Estes foram os principais fatores que levaram os participantes a considerar que, caso não sejam tomadas medidas para reter o conhecimento, a perda de conhecimento crítico poderá ser uma realidade num futuro próximo. Estas percepções estão refletidas nas afirmações expressas pelos participantes do focus group , a saber:

"Há processos de fabrico que se aprendem, mas não se ensinam. (...) Todos estes especialistas (...) são pessoas que têm hoje entre os 55 e os 60 anos e foram aprendizes durante $5,6,7,8,10$ anos. (...) É claro que em termos teóricos é possível transmitir o fenómeno que acontece durante [o processo], mas pôr isso em prática só fazendo."

"Não existe uma formação de base - que hoje é possível fazer-se, por exemplo, em sala - e depois fazer-se o desenvolvimento [na empresa]. E como não existe leva uns 10 anos a fazer [referindo-se à formação dos profissionais].”

A ameaça de perda de conhecimento é igualmente percecionada pelos colaboradores que estão mais próximos da idade de reforma. As afirmações apresentadas abaixo ilustram a perceção existente entre os colaboradores seniores de que o seu conhecimento não é valorizado internamente. Este sentimento poderá também transformar-se num entrave à retenção dos colaboradores seniores (ARMSTRONG-STASSEN \& SCHLOSSER, 2011).

"Eles próprios [os colaboradores] queixam-se que o conhecimento deles vai ser perdido. E que o management não tem uma política de transferir o conhecimento dos que trabalham hoje cá para a nova geração."

"É um sentimento que eles têm: 'isto [referindo-se ao conhecimento] vai-se perder'."

"Esse [referindo-se a um colaborador em particular] é um dos que está sempre a dizer: "não põem aqui malta nova a aprender'."

O passo seguinte consistiu na identificação, relativamente aos trabalhadores próximos da idade de reforma, de quem eram os colaboradores considerados chave para a organização. A sinalização das pessoas nucleares pressupôs a identificação do conhecimento crítico para os processos de fabrico que estas pessoas detêm e que as diferencia das restantes (p.e., o corte térmico ou a análise de desenho e aproveitamento de materiais). Incluiu também a identificação das competências soft que poderão ser determinantes para implementar algumas iniciativas de partilha de conhecimento (p.e., a predisposição para ensinar e transmitir conhecimento (p.e., "é a pessoa indicada para ensinar alguém”).

No sentido de obter uma noção mais clara sobre a dimensão do problema da perda de conhecimento, foram identificados quais os impactes organizacionais que estariam associados à saída dos perfis A e B. Os resultados observados corroboram, em grande medida, a visão de DELONG (2004) e de STRACK e colegas (2008), entre outros autores, tendo sido estabelecida uma relação direta entre a perda de conhecimento crítico a uma diminuição das capacidades organizacionais. A afirmação seguinte ilustra como a perda de conhecimento pode afetar a capacidade de produção de um determinado produto: 
"Daqui a 5 anos (ou quando estas pessoas se reformarem), porque não vai dar tempo para formar outras, deixamos de poder fazer [o] produto X, por exemplo."

\section{b) Próximos passos na implementação da estratégia de retenção.}

Conhecido e avaliado o risco de perda de conhecimento, importa retomar a questão central que motivou o projeto em curso: como reter o conhecimento crítico na unidade fabril? Seguidamente, serão elencadas algumas das iniciativas previstas, as quais expressam as restantes componentes que integram o modelo conceptual proposto (representado na figura 1), a saber: infra-estrutura organizacional para a retenção de conhecimento; iniciativas de partilha e armazenamento de conhecimento; e políticas de desenvolvimento e retenção de pessoas.

\section{$\underline{\text { Infra-estrutura organizacional de retenção de conhecimento }}$}

As questões relacionadas com a necessidade de promover um ambiente organizacional "amigo da retenção do conhecimento", assente em relações de confiança e que incentive a procura e reutilização de conhecimento, foram as menos evidenciadas no decorrer do focus group. Ainda assim, com o objetivo de promover de um contexto organizacional favorável à retenção de conhecimento e, sobretudo, alertar os colaboradores para a importância organizacional da GC está prevista a seguinte ação:

$\checkmark$ Divulgação e comunicação interna do projeto em curso: realizar reuniões com os colaboradores chave identificados (seniores), no sentido de divulgar o projeto e seus objetivos, evidenciando-se a criticidade do mesmo e a importância do envolvimento e contributo destes colaboradores.

\section{$\underline{\text { Iniciativas de partilha e armazenamento de conhecimento }}$}

Analisados os resultados, os responsáveis da unidade fabril privilegiaram as estratégias de personalização do conhecimento como forma de partilhar e reter o conhecimento:

"Reter o conhecimento que existe. Reter como? Só o consegues reter, se ele [colaborador] o passar para outra pessoa."

"As pessoas aprendem por ver e por comparação. (...) [referência a um episódio de visita à fabrica] Está ali um individuo que é mais jovem, é ele que está a fazer o trabalho, mas está a ser conduzido pelo outro [referindo-se a um colaborador mais sénior]."

"Temos que arranjar um grupo de rapazes que tenha uma formação tecnológica de base (do ensino secundário). (...) E essas pessoas têm que trabalhar ao lado dos outros [colaboradores seniores]."

"Existia um indivíduo (...) que deixou alguns escritos sobre caldeiraria (...) que podiam servir de base para aulas teóricas em sala. Só que, como nós sabemos todos, estas profissões aprendem-se com a prática. A pessoa tem que estar ali a assistir para fazer."

Com o propósito de incentivar a partilha de conhecimento entre gerações estão previstas as seguintes ações: 
$\checkmark$ Formação em posto de trabalho: elaborar planos de formação que permitam aos colaboradores juniores "aprender experimentado".

$\checkmark$ Mentoring/Formalização da figura de mentor: Identificar entre os colaboradores seniores um conjunto de mentores que possam acompanhar os aprendizes no dia-a-dia de trabalho.

Apesar da personalização ter sido mais evidenciada durante a sessão com os responsáveis, o road map do projeto de implementação da estratégia de retenção de conhecimento inclui, também, iniciativas que visam a explicitação do conhecimento. $\mathrm{O}$ que de resto está em linha com os estudos empíricos onde se verificou que a de explicitação/ codificação de conhecimento teve efeitos positivos na memória organizacional (FIEDLER \& WELPE, 2010) e, consequentemente, na retenção de conhecimento. No caso da unidade fabril em estudo existem um conjunto competências e conhecimento críticos que poderão ser formalizados e armazenados para que possam ser (re)utilizados por toda a organização. Nesse sentido, prevê-se a implementação das seguintes iniciativas:

$\checkmark$ Criação de uma biblioteca de conhecimento: Elaborar manuais de fácil consulta e acessíveis a todos os colaboradores. Implica recolher e compilar informação detalhada sobre processos, produtos fabricados atualmente, bem como os que se perspetivam fabricar num futuro próximo.

$\checkmark$ Criação de um repositório fotográfico e vídeo: Capturar imagens de operadores a desempenhar os processos de fabrico (p.e. desempanagem de chapas), registando-se assim o desempenho desses processos "passo-a-passo".

$\checkmark$ Identificação de experts (sinalizados pelos responsáveis durante o focus group): Afixar nos boards informativos - aos quais todos os colaboradores têm acesso - quem são os experts nos vários domínios de conhecimento e a quem os restantes colaboradores poderão recorrer para esclarecer dúvidas, pedir conselhos, etc.

$\underline{\text { Políticas de desenvolvimento e retenção de pessoas. }}$

Os resultados observados no presente estudo corroboram o argumento de que uma estratégia de retenção de conhecimento deverá ser acompanhada por iniciativas relacionadas com a gestão de pessoas (DELONG, 2004; LIEBOWITZ, 2009). As afirmações seguintes evidenciam a necessidade de desenvolver uma política, com enfoque no longo prazo, como forma de motivar, desenvolver e reter os colaboradores seniores e juniores:

\footnotetext{
"As pessoas estão desmotivadas para passar conhecimento, porque não se sentem reconhecidas."

“ (...) tem que se dar um plano [de evolução], a longo prazo, a essas pessoas. Não só técnica, mas também de regalias dentro da empresa. Entre elas as regalias salariais, porque eles são aliciados a trabalhar 'lá fora' [referindo-se à concorrência] (...)."

"Resta (ainda) identificar quem é que quer aprender e o que se vai fazer para manter os trabalhadores na empresa, à medida que vão adquirindo as competências."
} 
"O que não pode acontecer, se a empresa quer admitir pessoas não têm experiência, é serem "lançados aos bichos". Tem que haver algum seguimento e algum apoio às pessoas (...)."

“ $(\ldots)$ as pessoas sentem-se motivadas por serem contratados para essas funções [referindo-se à permanência dos colaboradores reformados como mentores]. Sentem que o management reconhece neles conhecimentos técnicos para o fazer."

No sentido de implementar e/ou reformular algumas práticas de gestão de pessoas que influenciam por um lado, a motivação para a partilha de conhecimento e, por outro lado, a retenção das pessoas na organização, foram planeadas as ações que se apresentam seguidamente:

$\checkmark$ Recrutamento e seleção de aprendizes: Iniciar um processo de recrutamento e seleção (R\&S) de colaboradores juniores - aprendizes - que sejam devidamente acompanhados pelos membros os mais experientes.

$\checkmark$ Desenvolvimento de competências: Combinar a aprendizagem em posto de trabalho com outras tipologias de formação que possibilitem a aquisição/ desenvolvimento de competências e conhecimentos técnicos (p.e. saber ler desenho técnico, conhecimentos de geometria, conhecer os símbolos e posições de soldadura, etc.).

$\checkmark$ Parcerias com escolas/ instituições de formação: Desenvolver conteúdos de formação, em parceria com outras instituições, para dar resposta às especificidades da unidade fabril.

$\checkmark$ Avaliação e desenvolvimento de competências: Definir uma matriz de competências onde estejam refletidas as competências e os conhecimentos identificados como críticos para a concretização dos processos de fabrico.

$\checkmark$ Identificação de sucessores: Identificar dentro da organização, ou com recurso ao R\&S, potenciais substitutos para os colaboradores nucleares sinalizados pelos responsáveis e que se encontram próximos da idade de reforma.

$\checkmark$ Planeamento de carreiras: Propor à Direção a criação e divulgação de planos de carreira para os perfis $\mathrm{A}$ e $\mathrm{B}$, onde se explicitem as oportunidades de evolução de acordo com as várias categorias profissionais (ex. aprendiz, trabalhador qualificado, etc.).

$\checkmark$ Manutenção da base de dados de experts seniores: Atualizar regularmente a lista de colaboradores experts nos vários domínios, incluindo-se colaboradores no ativo e reformados.

$\checkmark$ Reconhecimento dos colaboradores: Reconhecer (publicamente) o valor dos conhecimentos e competências dos colaboradores seniores, nomeadamente através da sua nomeação para papéis de mentor ou formador interno.

\section{CONCLUSÕES}

O envelhecimento da população ativa começa a estar amplamente presente tanto na agenda da GC, como na de GRH. Deste modo, perante a ameaça, mais ou menos real, de perda de conhecimento uma das questões centrais que se coloca é, certamente, a de saber 
como reter o conhecimento organizacional crítico para a concretização das metas organizacionais. Apesar dos estudos efetuados sobre esta temática, não encontrámos estudos empíricos onde se analisasse o processo de formulação e implementação de uma estratégia com este fim. O principal contributo do presente estudo foi, exatamente, analisar a fase inicial de um projeto organizacional lançado para reter o conhecimento dos blue collars. A análise deste processo permite-nos retirar algumas conclusões acerca da formulação de uma estratégia de retenção de conhecimento.

Em primeiro lugar, a decisão de desencadear iniciativas que visem reter o conhecimento organizacional crítico varia muito de organização para organização, dependendo fundamentalmente da forma como esse problema é percecionado internamente (DELONG \& DAVENPORT, 2003). No caso ALSTOM Portugal, a nossa análise indica que a média de idades dos blue collars funcionou como uma espécie de "alarme" que alertou a organização para a necessidade de reter o conhecimento crítico destes colaboradores. Conhecimento este que foi construído ao longo de vários anos de trabalho na empresa e que inclui além da expertise técnica, o domínio dos processos de fabrico, o manuseamento das máquinas e um conhecimento profundo dos produtos da empresa. $\mathrm{O}$ conhecimento dos blue collars identificados como colaboradores-chave envolve uma combinação de know-how, competências e conhecimentos técnicos que não é facilmente replicável, dificultando a possibilidade de substituir estes colaboradores num curto espaço de tempo. Situação que, na opinião dos membros da organização, torna a retenção de conhecimento uma necessidade urgente, uma vez que serão necessários alguns anos para formar os substitutos.

Em relação ao processo de identificação do risco de perda de conhecimento, propriamente dito, o recurso a uma metodologia qualitativa - através da entrevista em grupo permitiu recolher informação bastante mais detalhada do que seria possível utilizando algumas das métricas quantitativas sugeridas na literatura (e.g. LIEBOWITZ, 2009). Em particular, permitiu que os participantes refletissem em conjunto sobre o problema da perda de conhecimento na unidade fabril, expressando as suas perceções e opiniões, bem como fornecendo algumas pistas para a conceção de iniciativas de retenção de conhecimento ajustadas ao contexto organizacional em estudo.

Um terceiro aspeto a realçar prende-se com o planeamento dos próximos passos no sentido da implementação da estratégia. A seleção das iniciativas que visem promover um ambiente "amigo da retenção do conhecimento", bem como as iniciativas no âmbito da GC e GRH depende fundamentalmente da história e cultura organizacional. No caso ALSTOM 
Portugal, ficou evidente a preferência pela seleção de iniciativas que promovam o fluxo de conhecimento entre os agentes envolvidos - colaboradores seniores e juniores. A relação mentor-aprendiz surgiu, por isso, diversas vezes mencionada enquanto forma de promover a comunicação intergeracional, de reconhecer internamente o valor do conhecimento distintivo dos seniores, bem como de promover a partilha de conhecimento intergeracional.

Para concluir, importa referir que apesar de termos analisado um projeto em curso consideramos que a experiência desta empresa, não sendo suscetível de replicação, revelou algumas pistas relevntes para a formulação de uma estratégia que minimize o risco de perda de conhecimento crítico. Em termos de prática gestionária, a principal implicação deste estudo relaciona-se com a necessidade de conceber uma estratégia com enfoque no longo prazo, combinando os aspetos culturais, relacionais e estruturais associados à retenção do conhecimento.

Como qualquer investigação, este estudo apresenta também algumas limitações. Em primeiro lugar, o facto de se tratar de um estudo de caso o que impossibilita a generalização dos resultados. Uma segunda limitação desta investigação relaciona-se com a utilização utilização do focus group. Embora este permita captar a interação e as atitudes (e experiências) dos participantes, importa referir que estas ocorrem num contexto artificial (MORGAN \& SPANISH, 1984). Tentou-se minimizar esta limitação complementando a recolha de dados com análise documental. Acresce ainda que uma das investigadoras é colaboradora da ALSTOM Portugal, situação pode ter condicionado, de alguma forma, o distanciamento necessário entre o investigador e o seu objeto de estudo.

Finalmente, o facto do projeto de implementação da estratégia de retenção de conhecimento se encontrar, ainda, em curso não permitiu avaliar a eficácia da abordagem seguida pela empresa para evitar a perda de conhecimento na unidade fabril em estudo. Consequentemente, seria interessante realizar, dentro de dois ou três anos, um estudo semelhante, no sentido de perceber se as iniciativas planeadas geraram os efeitos desejados e se o risco da perda de conhecimento crítico foi minimizado.

\section{REFERÊNCIAS}

APPELBAUM, S. H., BENYO, C., GUNKEL, H., RAMADAN, S., SAKKAL, F., \& WOLFF, D. Transferring corporate knowledge via succession planning: analysis and 
solutions - Part 2. Industrial and Commercial Training, 44(7), 2012, p.379-388. doi:10.1108/00197851211267956

APPELBAUM, S. H., GUNKEL, H., BENYO, C., RAMADAN, S., SAKKAL, F., \& WOLFF, D. Transferring corporate knowledge via succession planning: analysis and solutions - Part 1. Industrial and Commercial Training, 44(5), p.281-289, 2012. doi:10.1108/00197851211245031

ARMSTRONG-STASSEN, M., \& SCHLOSSER, F. Perceived organizational membership and the retention of older workers. Journal of Organizational Behavior, (32), p.319-344, 2011. doi:10.1002/job

ASHWORTH, M. J. Preserving knowledge legacies: workforce aging, turnover and human resource issues in the US electric power industry. The International Journal of Human Resource Management, 17(9), p1659-1688, 2006. doi:10.1080/09585190600878600

BERGAMASCHI, M., \& URBINA, L. Modelos e Sistemas de Gestão de Conhecimento. In Anais do $1^{\circ}$ Encontro de Iniciação Científica e Pós-Graduação do ITA - XV ENCITA / 2009. São José dos Campos, SP, Brasil: Instituto Tecnológico de Aeronáutica, 2009.

CABRERA, Á., COLLINS, W. C., \& SALGADO, J. F. Determinants of individual engagement in knowledge sharing. The International Journal of Human Resource Management, 17(2), 2006, p-245-264. doi:10.1080/09585190500404614

CATTANI, G., DUNBAR, R. L. M., \& SHAPIRA, Z. Value Creation and Knowledge Loss : The Case of Cremonese Stringed Instruments. Organization Science, 24(3), p.813-830, 2013.

CEN, (European Committee for Standardization). CWA 14924-1: European Guide to good Practice in Knowledge Management - Part 1: Knowledge Management Framework, 2004.

CHRISTENSEN, P. H. Knowledge sharing: moving away from the obsession with best practices. Journal of Knowledge Management, 11(1), p.36-47, 2007.

doi:10.1108/13673270710728222

COHEN, W. M., \& LEVINTHAL, D. A. Absorptive Capacity: A New Perspective on Learning and Innovation. Administrative Science Quartely, 35(1), 128-152, 1990.

DELONG, D. W. Better Practices for Retaining Organizational Knowledge : Leading Edge. Reseach report, Accenture Institute for Strategic Change, 2002.

DELONG, D. W., \& DAVENPORT, T. Better practices for retaining organizational knowledge: Lessons from the leading edge. Employment Relations Today, 30(3), p.51-63, 2003. doi:10.1002/ert.10098

DELONG, D. W., \& FAHEY, L. Diagnosing cultural barriers to knowledge management. Academy of Management Executive, 14(4), p.113-127, 2000.

DELONG, D. W. (2004). Lost Knowledge: Confronting the Threat of an Aging Workforce (p. 272). New York: Oxford University Press. 
FIEDLER, M., \& WELPE, I. How do organizations remember? The influence of organizational structure on organizational memory. Organization Studies, 31(4), p.381-407, 2010. doi:10.1177/0170840609347052

HANSEN, M T, NOHRIA, N., \& TIERNEY, T. What's your strategy for managing knowledge? Harvard business review, 77(2), p.106-116,1999.

HOLAN, P. M. DE, \& PHILLIPS, N. Remembrance of Things Past? The Dynamics of Organizational Forgetting. Management Science, 50(11), p.1603-1613, 2004. doi:10.1287/mnsc.1040.0273

IPE, M. Knowledge Sharing in Organizations: A Conceptual Framework. Human Resource Development Review, 2(4), p.337-359, 2003. doi:10.1177/1534484303257985

LIEBOWITZ, J. Knowledge Retention: Strategies and Solutions. New York: CRC Press, 2009.

MARTINS, E. C., \& MEYER, H. W. J. Organizational and behavioral factors that influence knowledge retention. Journal of Knowledge Management, 16(1), p.77-96, 2012. doi:10.1108/13673271211198954

MARTINS, J. M. Gestão do Conhecimento: Criação e Transferência de conhecimento ( $1^{\text {a }}$ Edição., p. 116). Lisboa: Sílabo, 2010.

MASSINGHAM, P. Measuring the Impact of Knowledge Loss: More Than Ripples on a Pond? Management Learning, 39(5), p.541-560, 2008.. doi:10.1177/1350507608096040

MCDERMOTT, R., \& O'DELL, C. Overcoming cultural barriers to sharing knowledge. Journal of Knowledge Management, 5(1), p.76-85, 2001.

doi:10.1108/13673270110384428

MINBAEVA, D., FOSS, N., \& SNELL, S. Guest editors' introduction: bringing the knowledge perspective into hrm, 48(4), 477-483, 2009. doi:10.1002/hrm

MORGAN, D. L., \& SPANISH, M. T. Focus groups: A new tool for qualitative research. Qualitative Sociology, 7(3), p.253-270, 1984.. doi:10.1007/BF00987314

NAHAPIET, J., \& GHOSHAL, S. Social Capital, Intellectual Capital, and the Organizational Advantage. The Academy of Management Review, 23(2), 242, 1998. doi:10.2307/259373

NONAKA, I., \& KONNO, N. The Concept of "Ba": Building a foundation for knowledge creation. California Management Review, 40(3), p.40-54, 1998.

PARISE, Salvatore; CROSS, Rob; DAVENPORT, Thomas H. Strategies for preventing a knowledge-loss crisis. MIT Sloan Management Review, 47. p.4: 31, 2006.

RAO, M. Knowledge Management Tools and Techniques. (M. Rao, Ed.) (p. 456). Oxford: Elsevier Butterworth-Heinemann, 2005.

RIEGE, A. Three-dozen knowledge-sharing barriers managers must consider. Journal of Knowledge Management, 9(3), p.18-35, 2005. doi:10.1108/13673270510602746 
SANTOS, M. J., WANE, R., \& LOPES, O. Knowledge Sharing: Social, Cultural and Structural Enabling Factors. In C. Machado \& J. P. Davim (Eds.), Transfer and Management of Knowledge. London: Wiley-ISTE, 2014.

SCHMITT, A., BORZILLO, S., \& PROBST, G. Don't let knowledge walk away: Knowledge retention during employee downsizing. Management Learning, 43(1), p.53-74, 2011. doi:10.1177/1350507611411630

STRACK, R., BAIER, J., \& FAHLANDER, A. Managing demographic risk. Harvard business review, 86(2), 119-28, 138, 2008.

SUN, P. Y.-T., \& SCOTT, J. L. An investigation of barriers to knowledge transfer. Journal of Knowledge Management, 9(2), p.75-90, 2005. doi:10.1108/13673270510590236

WANE, R., \& SANTOS, M. J. People, Knowledge and Technology: Connecting the Dots from a Social Perspective. In C. Machado \& J. P. Davim (Eds.), Human Resource Management and Technological Challenges (pp. 69-97). Springer International Publishing, 2014. doi:10.1007/978-3-319-02618-3_4

WRIGHT, P. M., DUNFORD, B. B., \& SNELL, S. A. Human Resources and the Resource Based View of the Firm. Journal of Management, 27(6), p.701-721, 2001).

YIN, R. K. Case Study Research: Design and Methods (Third Edit., p. 181). London: SAGE Publications, 2003. 